\title{
Rheumatic fever and rheumatic heart disease: Where are we now in South Africa?
}

\section{Mark E. Engel*, Lies Zühlke ${ }^{\#}$ and Kate Robertson+ \\ *Research Fellow, Department of Medicine, Health Sciences Faculty, University of Cape Town \\ \#Paediatric Cardiologist, Western Cape Pediatric Cardiac Services Red Cross and Tygerberg Hospitals, University of Cape Town \\ +Visiting Research Fellow, Department of Medicine, Health Sciences Faculty, University of Cape Town}

\section{Address for correspondence:}

Dept of Medicine, J47-92

Faculty of Health Sciences, University of Cape Town

Old Main Building, Groote Schuur Hospital

Observatory

7925

South Africa

Email:

mark.engel@uct.ac.za

\section{ABSTRACT}

South Africa continues to face unacceptably high rates of rheumatic fever (RF) and rheumatic heart disease (RHD), despite readily available and inexpensive preventive measures. However, in the past several years, key players in South Africa's healthcare and political realms in addition to key players from many African nations have come together to acknowledge the persistent health burden attributable to RF/RHD and have agreed to a pledge of action to reduce it. The plan of action is a comprehensive RF/RHD prevention and treatment programme known as ASAP. The ASAP programme targets efforts to raise Awareness, establish Surveillance systems, Advocate for increased resources for treatment, and to promote Prevention strategies. South Africa currently has a demonstration site where activities in all of these key areas are currently underway. Efforts in the area of surveillance include a RHD prevalence study that aims to screen $\mathbf{4 0 0 0}$ school-aged children through the use of a mobile echo-surveillance unit. In addition to local efforts, South Africa will join an international initiative to create a global RHD registry that will aid in all aspects of prevention and treatment to further reduce the burden of disease attributable to RF/RHD. SAHeart 2009; 6:20-23

The ASAP programme collaborates with other similar programmes of the World Heart Federation (WHF) globally, and has also been adopted by the Inter Academy Medical Panel as one of six global programmes.

Following the Drakensberg meeting, there has been renewed interest among cardiologists, other medical and public health professionals, the community and most importantly, government to be proactive in combating RF and RHD.

\section{Scale of RF/RHD}

Over the last 150 years the developed world has experienced a dramatic decline in the incidence and prevalence of RF/RHD through improved living conditions and the widespread use of penicillin for the treatment of group A beta haemolytic streptococcal pharyngitis. However, developing countries, which account for $80 \%$ of the world's population, continue to face unacceptably 
high rates of RF/RHD, making it the most common cardiovascular disease affecting children and young adults in the world. ${ }^{(2)}$

Together, RF \& RHD affect about 15.6 million people worldwide, 2.4 million of whom are children between five and fourteen years old in developing countries. (3) Sub-Saharan Africa and South Central Asia account for the majority of cases. Almost 500000 new cases are declared every year. RF/RHD leads to an estimated 350000 deaths annually, and hundreds of thousands of survivors are left disabled without access to the expensive medical and surgical care that the disease requires.

It is estimated that $0.3-3 \%$ of those with untreated group $A$ beta haemolytic streptococcal infection progress to develop acute rheumatic fever; and such infection may often be asymptomatic. Approximately 40 - 60\% of episodes of RF result in RHD.

Africa, which contains $10 \%$ of the world's population, has a disproportionately high share of people living with RF/RHD. In 2002, of the 2.4 million children with RF/RHD living in developing countries, nearly half ( $>$ I million) live in sub-Saharan Africa, making the continent the major hotspot of RF/RHD. (3) RF is a disease of poverty; it is particularly rampant in low-income, overcrowded communities with poor housing conditions, poor nutrition and inadequate health services. A neglected "strep" throat (sore throat caused by streptococcal bacteria) can lead to repeated episodes of RF affecting mainly the large joints (arthritis) and the heart (carditis), causing chronic heart valve damage (RHD) which may necessitate expensive heart valve surgery. The condition is usually fatal if damaged heart valves are not repaired or replaced by major open-heart surgery.

RF and RHD are among the commonest causes of cardiovascular morbidity and mortality in developing countries. RHD is the second common cause of heart failure (after hypertension) in Africans and the commonest indication for cardiac surgery in tropical Africa, accounting for nearly $60 \%$ of open heart surgery. ${ }^{(4)}$

There is a paucity of information on the prevalence of RHD in South Africa. Two previous studies of the prevalence of RHD in South Africa used clinical auscultation (not echocardiography) to screen for RHD.(5.6) Both studies provided an estimate of 6.9// 000 school children, which may however, be an underestimate of the prevalence of RHD among children because of the low sensitivity of the method of screening used (i.e. clinical examination) and probable absence from school of more sick children with RHD. Clearly, alternative methods of screening for RF/RHD such as mobile echocardiography would provide a more accurate estimate of the prevalence of RF/RHD in the general population. A systematic review of prevalence studies found exceptionally high rates of RHD in sub-Saharan Africa, with the highest level in Kinshasa, DRC at 14/1000 school-aged children. ${ }^{(7)}$ Similar high prevalence rates have been found in Cairo, Egypt (5.1/I 000) and Addis Ababa, Ethiopia (6.4// 000). ${ }^{\left({ }^{8}\right)}$ More recently, an echocardiographic-based study in Mozambique reported rates of $30 / 1$ 000.(9)

To date there have been no studies of the incidence of RF/RHD in Africa. Anecdotal information suggests that the incidence of RF remains quite high in South Africa and that RHD is still the leading cause of acquired heart disease in children and young adults. ${ }^{(10)}$ In a 2002 report from a pediatric cardiology conference, a consensus opinion was that South Africa was in the midst of an RF epidemic ${ }^{(1)}$ despite national guidelines and recommendations for antibiotic prevention of the disease. ${ }^{(12)}$ Furthermore, there appears to be underreporting of RF cases by health care professionals, and poor administration of the RF notification system. A detailed analysis of the number of RF cases reported at hospital, municipal and provincial level for a 5-year period suggested underreporting by health care professionals and poor administration of the notification system.(13)

\section{Prevention / therapeutic options}

RF/RHD is a disease of poverty, of children, unrelated to lifestyle and killing young people in the prime productive years of their lives. Sadly, it is almost entirely preventable using demonstrated strategies that are cheap and cost-effective. Prevention efforts are most effective when supported by a comprehensive programme that includes health education for parents, children, teachers and health care providers, routine screening, availability of penicillin and maintenance of a disease registry to manage known cases. Moreover, primary and secondary prevention programmes can be implemented through the existing health care services without major additional costs.

In 1997, the South African Department of Health $(\mathrm{DOH})$ released a set of national guidelines on the primary prevention and prophylaxis of RF and RHD. The guidelines were intended to facilitate a comprehensive programme for the primary and secondary prevention of RF. Targeting health workers at the primary care level, they cover interventions addressing education, living conditions, diagnosis, treatment, referral, notification, and follow- 
up. An evaluation of adherence to the guidelines indicated that in addition to physician awareness and compliance with the national guideline being unsatisfactory, the method for case detection of RF was unclear. ${ }^{(12)}$ A revision of the guidelines has recently been submitted to the department of health and is awaiting approval.

Many first episodes of RF can be prevented by a simple injection of penicillin for strep sore throat (primary prevention). ${ }^{(10)}$ Even if the first episode of RF is not prevented, further episodes (which almost always lead to RHD) are entirely preventable through secondary prevention. Secondary prevention requires the delivery of regular penicillin injections to prevent further RF and worsening $\mathrm{RHD}$, and is the mainstay of controlling RF/RHD. ${ }^{(14)}$ At the end of the spectrum, is the need for surgical intervention to repair scarred valves. In 2000, the average cost of operative treatment for RHD in Africa was around US\$5 000; in low income countries of sub-Saharan Africa with a GNP per capita of less than US\$500, such as Ghana, increasing health expenditure to address this cost would adversely affect poverty reduction strategies. ${ }^{(4)}$ Unfortunately, the downscaling of quotas for operations over the years makes it difficult to establish the true extent of the need for this procedure in South Africa.

Vaccines to prevent rheumatic fever?

The development of vaccines would represent the most ideal strategy of control to prevent group A streptococcal-related disease. Despite attempts dating back for more than 50 years, vaccination has hitherto proved impossible. Recently, concentration of efforts has been on the organism's M Protein epitopes, the diversity of which need to be elucidated: a study is currently underway to establish the molecular epidemiology of the bacteria in Cape Town. It is thus conceivable that South Africa would play a pivotal part in clinical trials of new vaccines for group $A$ streptococcus in the future.

\section{Lack of attention}

There has been a dearth of activity related to RHD over the past two decades in Africa and elsewhere as the attention of cardiologists has tended to focus on ischaemic heart disease, while the attention of public health practitioners has tended to focus on HIV, AIDS and TB. Hypertension, and its cardiac and cerebrovascular sequelae probably represent the major burden of cardiovascular disease in Africa, but RF/RHD is still the major cause of acquired heart disease in Africa and indeed the world. ${ }^{(15)}$ It is amongst the top three causes of heart failure in sub-Saharan Africa. ${ }^{(16)}$

\section{CURRENT INITIATIVES}

Launch of South African demonstration site

In South Africa, the ASAP Programme has designated the Vanguard Community in Cape Town as its demonstration site. To date, the ASAP team has been conducting awarenessraising presentations amongst parents, teachers and community members. In addition, we have held seminars for health professionals to enable them to recognise and diagnose streptococcal sore throat, and make valid management decisions.

Recently, portable echocardiography had been applied in landmark papers to assess the incidence of RHD in Cambodia, Mozambique ${ }^{(9)}$ and Tonga. ${ }^{(17)}$ It is well known that Doppler echocardiography is highly sensitive in demonstrating valvular regurgitation. ${ }^{(18)}$ Using this method, the highest incidence of RHD hitherto has been demonstrated in Tonga. ${ }^{(17)}$ In addition, their findings show that auscultatory findings alone would have missed more than half of the pathological lesions as later defined by echocardiography. This obviously has great implications for a screening programme based in an area of high-prevalence, with little or no resources.

The recent acquisition of a customised mobile echo-surveillance unit has enabled the launch of the surveillance component of the programme, where approximately 4000 school-aged participants will be screened for the prevalence of RHD. This involves a comprehensive assessment with particular attention paid to skin lesions, auscultation by a pediatric cardiologist followed by a full echocardiogram. This dataset forms part of a prospective study appraising echocardiography as a screening tool for RHD in developing countries. In addition, participants will be screened for skin conditions and referred to the appropriate clinic for follow-up. The skin assessment is also of interest following work from Australia looking at relative incidences of pharyngitis verses pyoderma in high-prevalence communities. ${ }^{(19)}$ All echocardiograms will be reviewed by a pediatric cardiologist and all cases detected will be reviewed by partners at Red Cross Hospital. It is anticipated that the results from this study, in addition to providing a comparison between auscultation and echocardiography in our setting, will add to the growing body of literature establishing the world-wide prevalence of RHD.

\section{Establishment of a global registry for RHD}

In 1972, the WHO launched a register-based programme to combat RF/RHD. Subsequently the programme was expanded and 
by 1990, registers had been established in 16 countries with over one million school-going children screened yielding over 3000 cases of RHD. However the declining rate of RHD in developed countries brought this initiative to a halt and in 200I, the $\mathrm{WHO}$ ceased its funding to this global programme.(20) Experience elsewhere has provided evidence of registers realising notable successes in reducing RF recurrence.(21) Thus, the absence of contemporary, systematically collected data is a gap that needs to be bridged in order to effectively treat patients with RHD in developing countries, where the rate of RF/RHD has not mirrored the decline in developed countries.

A global registry was launched at the South African Heart Association Congress 2008 and will be an international collaboration within the auspices of the WHF and ASAP. The purpose of this registry is to collect data on demographic and clinical profiles of patients with RHD in low and middle-income countries. It is also important to ascertain whether differences in patient characteristics in communities have clinical relevance especially as regards outcomes, since this may impact upon management strategies within different communities and age groups. A registry should also highlight deficiencies in service delivery to the most at risk patients and therefore guide in the initiation of more specific interventions. McDonald et al have suggested priority-based guidelines to evaluate and manage patients with severe, moderate and mild disease. ${ }^{(21)}$ In further assessment, specific performance indicators can then be reviewed in line with these guidelines. (22) The computerised register of cases of RF and RHD will be used to improve treatment adherence in order to prevent recurrent RF and the development of RHD necessitating surgery. In addition, the registry will serve as an epidemiological tool for gathering detailed echocardiography information for the purpose of research, compiling reference ranges and guiding the development of protocols and guidelines to improve secondary prophylaxis adherence. ${ }^{(23)}$

It is envisaged that the model of surveillance and prevention of RF/RHD will be successfully established, and can be rolled out at country level in order to prevent RF among children and thus avoid RHD and cardiac surgery. Through efforts such as ASAP as well as implementation of the National Department of Health guidelines for the prevention and treatment of RF/RHD, it is certainly feasible that both RF and RHD could be eradicated from South Africa within one or two generations, mimicking the achievements made recently in Cuba. ${ }^{(24)}$

\section{REFERENCES}

I. Mayosi B, Robertson K,Volmink J, et al.The Drakensberg declaration on the control of rheumatic fever and rheumatic heart disease in Africa. S Afr Med J 2006; 96: 246.

2. Carapetis JR, McDonald M,Wilson NJ.Acute rheumatic fever. Lancet 2005; 366: 155-68.

3. Carapetis JR, Steer AC, Mulholland EK, et al. The global burden of group A streptococcal diseases. Lancet Infect Dis 2005;5:685-94.

4. Amoah AG, Kallen C. Aetiology of heart failure as seen from a National Cardiac Referral Centre in Africa. Cardiology 2000;93:1 I-8.

5. McLaren MJ, Hawkins DM, Koornhof HJ, et al. Epidemiology of rheumatic heart disease in black school children of Soweto, Johannesburg. Br Med J 1975;3:474-8.

6. Maharaj B, Dyer RB, Leary WP, et al. Screening for rheumatic heart disease amongst black school children in Inanda, South Africa. JTrop Pediatr 1987;33: 60-I.

7. Steer AC, Carapetis JR, Nolan TM, et al. Systematic review of rheumatic heart disease prevalence in children in developing countries: the role of environmental factors. J Paediatr Child Health 2002;38:229-34.

8. WHO. Rheumatic fever and rheumatic heart disease: report of a $\mathrm{WHO}$ expert consultation, vol. Technical Report Series, No. 923, 2004.

9. Marijon E, Ou P, Celermajer DS et al. Prevalence of rheumatic heart disease detected by echocardiographic screening. N Engl J Med 2007;357:470-6.

10. Robertson KA, Volmink JA, Mayosi BM. Antibiotics for the primary prevention of acute rheumatic fever: a meta-analysis. BMC Cardiovasc Disord 2005;5: I I.

11. Department of Health SA. Modernisation of tertiary care services. Report on the paediatric cardiology workshop. Strand, South Africa, 26 - 27 September 2002. In: http://www.doh.gov.za/mts/reports/cardiology0 I.html (Last accessed 22///2009).

12. Robertson KA, Volmink JA, Mayosi BM. Lack of adherence to the national guidelines on the prevention of rheumatic fever. S Afr Med J 2005;95:52-6.

13. Nkgudi B, Robertson KA, Volmink J, et al. Notification of rheumatic fever in South Africa - evidence for underreporting by health care professionals and administrators. S Afr Med J 2006;96:206-8.

14. Manyemba J, Mayosi BM. Intramuscular penicillin is more effective than oral penicillin in secondary prevention of rheumatic fever: a systematic review. S Afr Med J 2003; 93:212-8.

15. Sliwa K, Wilkinson D, Hansen C, et al. Spectrum of heart disease and risk factors in a black urban population in South Africa (the Heart of Soweto Study): a cohort study. Lancet 2008;371:915-22.

16. Mayosi BM. Contemporary trends in the epidemiology and management of cardiomyopathy and pericarditis in sub-Saharan Africa. Heart 2007;93:1 1 76-83.

17. Carapetis JR, Hardy M, Fakakovikaetau T, et al. Evaluation of a screening protocol using auscultation and portable echocardiography to detect asymptomatic rheumatic heart disease in Tongan school children. Nat Clin Pract Cardiovasc Med 2008;5:41।-7.

18. Ozkutlu S, Hallioglu O, Ayabakan C. Evaluation of subclinical valvar disease in patients with rheumatic fever. Cardiol Young 2003; 1 3:495-9.

19. McDonald MI,Towers RJ, Andrews RM, et al. Low rates of streptococcal pharyngitis and high rates of pyoderma in Australian aboriginal communities where acute rheumatic fever is hyperendemic. Clin Infect Dis 2006;43:683-9.

20. Strategy for controlling rheumatic fever/rheumatic heart disease, with emphasis on primary prevention: memorandum from a joint WHO/ISFC meeting. Bull World Health Organ 1995;73:583-7.

21. McDonald M, Brown A, Noonan S, et al. Preventing recurrent rheumatic fever: the role of register-based programmes. Heart 2005;91:1 131-3.

22. Eissa $S$, Lee R, Binns $P$, et al. Assessment of a register-based rheumatic heart disease secondary prevention program in an Australian Aboriginal community. Aust N Z J Public Health 2005;29:521-5.

23. Stewart T, McDonald R, Currie B. Acute rheumatic fever: adherence to secondary prophylaxis and follow up of indigenous patients in the Katherine region of the Northern Territory. Aust J Rural Health 2007; 15:234-40.

24. Nordet P, Lopez R, Duenas A, et al. Prevention and control of rheumatic fever and rheumatic heart disease: the Cuban experience (1986-1996-2002). Cardiovasc J Afr 2008; 19:135-40 